\title{
On Algorithmic Opacity and Moral Certainty
}

\author{
Draft, November 2020
}

Oleg Litvinski

CIRST ('Centre interuniversitaire de recherché sur la science et la technologie')

UQAM ('Université du Québec à Montréal')

Montreal, Canada

Email: litvinski.oleg@courrier.uqam.ca

orcid.org/0000-0002-4564-7962

\begin{abstract}
In modern society, algorithms play an important role in social and cultural realms, in political and economic spheres. In spite of algorithmic pervasiveness in many areas and wide diffusion in digital life, algorithmic opacity is still poorly understood compared to other ethical issues (e.g., fairness, accountability, and transparency). In this essay, we try to elucidate the relation between algorithmic opacity and moral certainty from the individualistic standpoint and through the virtue ethic perspective. For doing so, we follow hermeneutic tradition and rely on interpretation of recent authors and relevant papers. We summarize our argument as follows: if the algorithm is understood as the combination of rules and numbers we create for simplifying our lives and sharing with others, then our present activities and future actions as imagined, realized or missed, ascertain if algorithmic opacity become a moral issue or problem for us and others. Among the implications, we emphasize that sometime dormant and hard to anticipate, algorithmic opacity becomes an apparent during executions, deployments and prolonged uses of algorithmic systems. Moreover, our lived experience and disharmony between our unrealized expectations and unanticipated algorithmic behavior may lead to moral issues and problems for us and others. Overall, algorithmic opacity may constantly evade the formalization efforts (e.g., outlining as guidelines, principles) or quantification exercises (e.g., assigning numerical values to symbols or signs), both of which are essentially social practices.
\end{abstract}

Keywords: algorithm, algorithmic opacity, moral certainty, virtue ethics, qualitative interpretative research, hermeneutics.

Note: the previous version of this paper was presented at EASST/4S Conference, virPrague, August $20^{\text {th }}$, 2020 (https://www.easst4s2020prague.org/) 


\section{Introduction}

Algorithms, their widespread diffusion and use raise many ethical questions. For example, algorithm and moral action, the right decision and avoidable mistake in the course of active engagement in thinking about future behavior and activities deserve, we consider, a proper moral investigation for clearly distinguishing between the 'should' do and the 'might have' deed. In other words, we try to illuminate in this essay a perplexing link between algorithmic opacity and moral certainty, a four words and concepts that attract an attention of researchers and practitioners in various domains and fields under closely related terms and concepts (i.e., black-box, understanding, meaning, and interpretation) and still, however, not properly articulated (Danaher 2019; Binns 2019; Wasilow and Thorpe 2019). They are, it seems to us, at the core of many moral tensions that are visible and audible during public deliberations and in scholarly circles touching upon digital economy, culture and social life, the future of the works as well as the place and role of human beings on this planet (Vallor 2017; Mittelstadt 2019; Green 2019).

This essay will also depart from a current tendency in contemporary scholarship and public conversation to overemphasize the moral agency (i.e., moral agent vs. moral patient duopoly) at the expense of the theme of moral sensitivity and attention to everyday behavior (Brundage 2014; Bogosian 2017; Misselhorn 2018; Herzog 2019). Matters of moral reflection are often reduced to a practical problem-solving and decision-making with the unfortunate emphasis on the application of understandable and universal rules, norms or principles to particular cases (Hoyos-Valdés 2018; Abend 
2019; Moulin-Stożek 2019; Abbott 2020). Moreover, this essay will weight in the consideration against the background of an ancient tradition which places at the heart of ethics the idea self-knowledge (i.e., the clearer understanding by removing obstacles, be it conceptual confusion or misplaced attitudes and habits). For doing so, we will adopt the point of view of a living subject, of a moral creature and emphasize a dialogic prose for articulating an argument according to the criteria of moral argument.

The main question we will try to elucidate in this essay concerns the relation between algorithmic opacity interpreted as the lighter version of the black-box problem (i.e., it may take form to various degrees of understanding of algorithmic functioning or its interpretation) leading to real action, on the one hand, and moral certainty on the other. In short, our argument is the following. If the algorithm is understood as rules and numbers, we follow for simplifying our activities in our lives and sharing with others, then our future actions and present behavior as imagined, realized, or missed, influence whether an algorithmic opacity becomes a moral issue, or problem, if at all, for us and others.

Methodologically speaking, this work follows qualitative research principles and practices in the tradition of interpretative hermeneutics, a kind of qualitative research when and where the focus is on interpretation by combining and contrasting various points of view, positions, arguments, and reasoning pertaining to investigator interests. This kind of approach enlightens a long-standing issues and problems by pointing out often forgotten and overlooked ways of seeing and considering perspectives (Roberge 2011; Tripathi 2017; Romele et al. 2020). 
For doing so, we situate first virtue ethic as distinct from consequentialist and deontological traditions in the next section. Second, we lay out and clarify the notion of an algorithm in the social realm. Third, we elaborate and interpret the opacity and its various kinds (i.e., epistemic, ontological, and practical). Fourth, we discuss how they are all situated in the regard to the moral certainty. Fifth, we detail the implications before, finally, summarizing the argument around actions, expectations and moral issues as well as positioning the essay in the larger constellation of literature.

\section{Background and Context}

Usually ethics and practice of ethics may be viewed as argumentation for doing a 'right thing' (i.e., a kind of persuasion about noble principles and actions) or showing standards and behavior (Russell 2013). In the latter case, we face the display, the exhibition and demonstration through which the ethical character or deed can be judged. Also, we need to be aware of the arising tension when and where standards and behavior come apart. Usually, this experience is the conflict between values expressed in standards and our desires and expectations, emotions and feelings.

In this essay, we follow a particular brand of applied ethics known as virtue ethic which is distinct from consequentialist and deontological approaches. In the essence, applied ethics aim to guide and describe actions and deeds (i.e., 'what' and 'ought', right vs. wrong, good vs. bad) when and where the concerns are about the moral judgment of human affairs in practical circumstances while normative and metaethics investigate the grounds and justifications of moral realm. 
Briefly summarized, virtue ethics are preoccupied with traits of character and consistent behavior rather than purposeful actions underpinned by reasoning in each particular case (e.g., be honest, charitable or generous and act accordingly). They are about the personal dispositions and character, socially perceived characteristics in the context of local or global practice and social life (Walker 1989; Bailey 2017; Curren 2019).

For virtue ethics, the moral value is the virtue itself which is worth to pursuit while in consequentialist and deontological traditions the moral value relates to the consequence of action or conformity to principles, respectively. For moral individual, the 'good' and 'right' is shaped in virtue ethics by reference to the virtue while in other two traditions, it refers to the utility principle (i.e., consequentialism) or intrinsic to the action (deontological ethics). For consequentialism, the value of action depends upon the aim (e.g., accepting a happiness or an absence of pain as the objective) while for deontological tradition the action is based upon an obligation, a duty to act (e.g., a calling or a devotion to a cause). However, it is hard to anticipate an action directly or indirectly (e.g., rulefollowing action) for the adepts of consequentialist tradition while universal obligations or duties are not always person-relative (i.e., an individual may choose to reject an absolute virtue of some kind). Finally, these traditions seem to assume the existence of social realm (i.e., groups) while virtue ethics does not.

Lastly, four criteria proposed by De Mesel $(2015,2018,153-172)$ serve us as guiding principles for aligning our interpretation with a proper moral argument. Firstly, the moral argument involves a participant attitude: being present, an active involvement without necessarily speaking or voicing an 
objection toward participants in the conversation. Secondly, this argument does not lead to sidelining of moral certainty (i.e., the conclusion of alleged argument does not entail the absence of certainty in extreme cases). Thirdly, moral thinking is not only about impending action per se (i.e., a particular situation and context involving a decision, choices, and a trigger to act). Lastly, a moral argument touches upon personal facets, namely individual traits as a human being, cognitive efforts to execute a task (i.e., 'thinking for yourself'), and its conclusion is not someone else conclusion (i.e., 'individual voice'). Failing to leave up to at least one of these criteria disqualifies the moral argument from being relevant to the problem under consideration.

\section{Algorithm}

In the social realm and personal matters, the concept of an algorithm encompasses usually two separate notions. On the one hand, the procedure, recipe and other similar terms are commonly associated with the rules and instructions, step-by-step guides (Totaro and Ninno 2014; Neyland 2015; Martignon 2015). On the other hand, notions of a sign, symbol and number are arranged as quantification and calculation or other mathematical operations (Chabert et al. 1999; Høyrup 2018).

Historically speaking, the notion of an algorithm occupies one of the central places in mathematics across continents and cultures (Chabert et al. 1999; Ogawa 2001; Sriram 2005). In this essay, however, we circumscribe the algorithm as studied, practiced, and transmitted in the social realm; also, we consider scholars in medieval universities as an appropriate starting point when the ideas of an algorithm and certainty may have intersected. In Information and Communication Technology circles, 
the notion of the algorithm is still debated issue. For example, algorithms as 'recursion' and algorithm as 'abstract state machine' are mainstream views in computer science (Vardi 2012). The closest to our position is the definition given by Hill (2016) who considers algorithm as combination of purpose, context, and description.

Speaking of the roles played by the algorithm in our lives, we argue that the simplification and sharing of objects and lived experiences are the most common. The first one, the simplification, consists of making complicated objects and tedious tasks simple and malleable ones. Faced with some challenges, difficult situations, the ones that demand from us a great deal of cognitive efforts and time, we naturally try to overcome these situations, to recognize obstacles and to avoid repeating the same work twice. So we create our own step-by-step guides and routines as a sequence of events, of actions and accompanied decisions we could repeat, the choices we face in the future and unforeseen circumstances. Once encoded in the rules and numbers, our mental and physical efforts end up as recipes to follow with the ease and without wasting precious attention or other cognitive resources.

The second one, the sharing or shareable feature, facilitates the exchange, relations and interaction between ourselves and other social creatures. As such, we like to keep in touch with our neighbors and family and we have an urge to share our experiences, our decisions and choices with our close friends and relatives, with coworkers and neighbors. In doing so, repetition and proximity during face to face encounters function as a social bond. 
Combined together, our lives depend on rules and numbers for simplifying and sharing of our activities and experiences. An algorithm is best then viewed as personalized arrangement and not as a purely technological system filled with models and data, mechanisms and mathematical logic.

\section{Opacity: its Nature and Close Neighbors}

The opaque, vague and similarly sounding and linguistically close words make sense once situated in the appropriate vocabulary and terminology defining the furniture of the reality. Firstly, the opaque or vague in metaphysical sense are a proper notion and concepts to consider (Merricks 2001; Abasnezhad and Jenkins 2018). In this sense, the opacity or vagueness accompany the indeterminacy. Secondly, the opacity or vagueness in the epistemic sense is not under consideration while being wildly popular in the literature. Related to the claims about knowledge and propositions, this kind of opacity receives the most attention in literature during recent debates (Lynch 2014; Durán and Formanek 2018). This opacity may be understood as related to a priory knowledge (i.e., hinges, intuitions) and the genuine evidence or bona fide facts (Coliva 2016; Schönbaumsfeld 2016). In this sense, the term opacity is understood as the lighter version of the so-called black-box problem (i.e., the lack of understanding of algorithmic functioning and interpretation of its results (Mittelstadt et al. 2016; Finn 2019)). Sometimes, the opacity is mentioned alongside the transparency (Zerilli et al. 2019; Shin and Park 2019), accountability (Cath 2018; Espeland and Young 2019), privacy, equity and inequality (Nyholm 2018; Shadbolt et al. 2019, 149-200; Hagendorff and Wezel 2019), interpretability (Hansen and Krieger 2019), inscrutability (Kroll 2018). In most cases, the mention of algorithmic opacity involves decisionmaking, choices, consensus and agreements in the course of action and notions of community and 
involvement in its practice. To the same category, we also assign a so-called ontological term popularized in ICT and related disciplines (Poli and Obrst 2010; Guizzardi 2020) which is a kind of representational notion.

Moreover, we also exclude a particular kind of ontological opacity of the real world object as in our case we are interested only in the artificial ones. We consider this kind as the lighter version of ontological vagueness (Shapiro 2006; Tahko 2012). Thus, physical or material real world objects are ontologically vague or opaque to some degree (Åkerman 2012; Keil 2013; Weber 2013). For example, the cup on the table and the table are not naturally occurring phenomena. Although, both of them exist and are real, the slight change in the, for example, molecular structure may end up in other unrecognizable objects. This is a so-called border line case where a slight fluctuation of structural elements, be it object or its context, change the substance and form of an object. The same also apply to other facets and levels of reality (e.g., atom for physical entities, cell for living creatures). What is left is the kind of opacity that pertains to, in our opinion, routines and everyday practices where objects are essentially a kind of technological tools embedded in practices and routines. This kind of opacity relies mostly on the visual perception and aspect-seeing, experience and habit. For example, an object may be interpreted according to different criteria that are often related to the personal experience, long-standing practices and affiliations with some community (Feest, 2017; Malin 2019; Krzychała, 2019). The same may apply to other kinds of perception, namely, hearing, touch, smell and taste.

To sum up, the notions of action and activities at the nexus point between future and present, between imagined and occurred actions is the common element between these opacities. For this to happen, 
decisions, choices leading to actions are needed, but sustained cognitive efforts, such as reasoning, inference, and judgment, are not always required.

\section{Certainty and moral certainty}

Historical Landmarks and Contemporary Ramifications

A short historical sketch retracing and reinterpreting a shift in notions and perspectives helps us to appreciate how a split between a physical and humanistic sense of certainty may have occurred.

During medieval epoch, learned thinkers and scholars introduce in their writings the notion of certainty in the 14th century: Albert the Great speaks about moral certainty and intellectual life while John Buridan refines it (Saarinen 1993). The former distinguishes between an absolute moral certainty and moral ground of a particular action more or less based on a strong or weak opinion. For him, three kinds of certainty are available in practical matters: neither of two alternatives are acceptable, so look further; among two, the more affirmative and authoritative is preferred; and, lastly, a totally ambiguous situation. For his student, Buridan, the agent thought, decision-making process and conflicting reasons to act are all ingredients for further elaboration. Four situations than arise in judgment between practical cases A or B: (a) no reason for either A or B; (b) both cases are supported by comparably equal reasons; (c) the case A is stronger, but other reasons or justifications do support its counterpart; and (d), all doubts are absent supporting A over B (idem.,). The relatively straightforward parallel with 
contemporary epoch is simple: our moral judgments in practical affairs depend on and are constrained by finite reasons and justification.

In 1714, Bernoulli combines certainty with the notion of a chance while trying to articulate a mathematical paradox linked to the game and probabilities (Bernoulli et al. 2006). Four themes permeate the work of Bernoulli in regard to the notion of certainty in his lifelong efforts of the mathematization of social life and living beings. In contrast to the previous generations when certainty was a kind of expectation in civil and commercial spheres, the notion of chance delineates the certainty of events in the physical world. Secondly, a various degrees of certainty ranged from the highest to its opposite (i.e., no doubts at all vs. fully doubtful or sceptical). Thirdly, the complete (or perfect) certainty meant the observation of the infinite number of events which is combined with the mathematical operation based upon fixed and constant notions (e.g., counting). Lastly, the objective certainty was about past, present, and future things and their existence that are independent of our opinion or knowledge whereas the subjective certainty pertained to our knowledge about existence of such things (i.e., a probability is a degree of certainty). Moreover, moral certainty tended to approach as close as possible the complete certainty inasmuch as the difference is not perceivable. The notion of moral certainty resided in the necessity of contractual agreements and consensus (i.e., institutionalized), as well as personal beliefs (i.e., hypothetical cases constitute a difference between what is assumed to exist as a thing and its past, present, or future existence), while a physical necessity referred to the naturally occurring phenomena without a moral notion per se (idem.). Decades later, Buffon further refined certainty of the physical world and certainty of human affairs (i.e., "moral certainty") (Hey et al. 2010). Interested in measurement of certainty level as available evidence of observed effects, Buffon 
combined experimentation and mathematical notions and operations. According to him, the physical certainty was the most certain of all certainty whereas certainty per se was not an absolute concept. While a physical certainty was measured by repeated experiments during a long period of time, the sources of certainty in human affairs were an experience, analogy (i.e., an aggregate of relation of known things), and testimony (i.e., a second-hand opinion or a witness account). In regard to the relation between mathematical operations and practical matters, the moral person had taken into account benefits, risks, and hopes, while a mathematician executing a quantification may have dispensed with such prosaic affairs. Summing up, while Bernoulli enhanced a rich conceptual vocabulary around certainty with probability, necessity, and contingency, such constellation some decades later in the hands of Buffon formed a basis of mathematical operation applied to the newly articulated art of experimentation. This short conceptual archaeology helps us to discern where and when analogy and other heuristics may have led to granular versions of physical, social, and human dimensions of certainty ${ }^{1}$.

Inspired by Taylor (1980, 1985a, 1985b), we could draw some parallels between these epochs and contemporary literature mentioning uncertainty across various and disparate fields and domains, such as economics (Kessler 2008) and financial market (Beckert 2019), research management (Vertesi 2020), personal and family matters (Katz 2020) and sustainability (Diwekar et al. 2021). The conceptual vocabulary, grammar, and rules in previous centuries and now constitute, transform, and describe the social reality while being exposed to criticism and open to contestation. It is in the articulation from the point of view of an individual at a particular time and interactions with friends and

An interested reader may consult published in recent decades monographs thoroughly exploring certainty, such as Clark (2015), Kampourakis and McCain (2019). 
foes, acquaintances and close relatives that the social reality is constructed. This conceptual vocabulary also shapes the conduct and boundaries of the behavior of this particular person via acceptable or not norms, and the distinction between right and wrong action. Rules and agreements carve out what is considered normal or deviant and is compared against such consensus around practices deemed legitimate or inappropriate. The challenges originated in these practices facing a particular phenomenon and failing to meet them open door for other possibilities to be realized via the re-articulation of the previous version of acceptable conceptual vocabulary.

\section{Certainty: Kinds and Illustrations}

It is worth to describe the certainty and to distinguish its categories. Human beings face constantly certainty and doubt in the course of their everyday flow of actions regardless of circumstances in most cases. But what comes first? Certainty about something to doubt undeniably precedes the doubt which is the position we defend. Speaking of them, the idea about a concept or object, be it natural or an artificial, needs to be anchored in the mind before a doubt about such object or concept can spring from somewhere and doubt about can be even envisioned. A doubt about doubt, some may reply. But in such case, this someone denies the mere possibility of forming a term or a concept to doubt about later on. In the case of certainty, however, this is not the situation. Moreover, formulating certainty and placing it as first open doors for the possibility of other kinds, such as doubt. Moreover, we characterize certainty as some kind of "hinges" (Moyal-Sharrock 2004, 126-132; Moyal-Sharrock and Brenner, 2005). Usually, certainty is based on perception, sensing, and self-awareness, and even before being articulated, communicated, or even expressed. In other terms, someone in the state of certainty is not 
really paying attention to something, not being aware of, not requiring a direct and constant concentration or effort. The certainty in this sense seems to be a background on which other things are based.

At this stage of our elucidation, we need to clarify the kind of criteria for distinguishing between various certainties and choosing appropriate ones for us. Among recent works, we note two authors, O’Hara (2018) and Hermann (2015) whose works explore these kinds of criteria. O’Hara (2018, 6-17) elaborates four criteria, but his interpretation of "indubitability" ("impossibility of reasonable doubt") is, in our view, oriented toward reasoning and investigation; his "foundation" is mostly about collective and language; his "local and universal" criterion is geared toward a historical community; finally "logical structure" is the closest to our case, but still insufficient as the articulation of an argument is a kind of moral reasoning (i.e., it necessitates a cognitive effort and practice). In short, O’Hara (2018) criteria orient investigation toward community while our case is exclusively about the individual. Summing up, criteria proposed by O’Hara (2018), while closely related to the present case, are slightly different in focus and perspective if compared to those of Hermann (2015): the former are about others while the latter are mostly oriented on an individual and first-person view.

Hermann $(2015,86-87)$ proposes six criteria characterizing moral certainty, namely (1) the impossibility of a reasonable doubt; (2) the moral issue is widely accepted as such; (3) in some circumstances, insisting on doubt shows a lack of common sense; (4) mistakes or errors are impossible in principle; (5) supplemental reasons for achieving more certainty are unavailable; (6) the certain 
evade a (definitive) articulation in ordinary context. These criteria can guide us in deciding what is needed in the idealized cases that this or that feature of an algorithm could fulfill. Also, we will use imaginary figures Azeus and Begoa in order to illustrate discussed positions in each of sixth cases ${ }^{2}$.

\section{Doubt and future (first case)}

Let us examine and discuss under what circumstances a "reasonable doubt" about imagined future, whatever unrealistic or realistic, is possible at all.

Among two characteristics of an algorithm, the number is the most obvious to start with. Commonly understood as mathematical symbols and operations, numbers are the most sure and certain kinds of concept or object to come by. They are taught and learned since early childhood as arithmetic operations and practiced multiple times per day. Notwithstanding cultural, historical, or regional differences (e.g., Arabic and Latin numerical systems) and their interpretations, their meaning is the most basic belief across centuries, generations and continents. Moreover, numbers stay the same when and where they are in the future or in the present, but their interpretation may evolve. In short, if the algorithm is understood as numbers and only as manipulation of numbers, the moral certainty from first-person view would not be an issue or problem.

2 While Azeus plays a primarily role in our illustration, Begoa is confined to comparative status for clarifying the logic of our argument and our position. We would avoid the recourse to the metaphor as each one is grounded in some particular 15 context and easy to misinterpret (Ervas et al. 2018).

Litvinski On Algorithmic Opacity and Moral Certainty v3 
Speaking of the rule, we defend the position that the rule has been the social convention, often followed, always aspired to, sometime appropriately or not ignored. Even before considering this case, we need to clarify one issue with rules, understood as instructions or guidelines. We speak about a person, an individual and the question then become whether a private rule could possibly exist in principle. If there are any conditions that a person could create his or her own useful rule (e.g., a stepby-step guide), correctly applying and consistently using it without sharing or informing others? For the sake of the argument, we adopt the position that private rule may exist (Azzouni 2017, 73-85). Hence, our rules are sometimes to be shared and they are often convention and subject to constant change during negotiation. We leave aside the question of how a rule becomes a rule and the tension that shared rule is not applied unless it is changed. If the rule is shared, it may be negotiated and thus changed.

One other remark pertains to the tension between private and shared rule: if it is private it is not shared, but, once shared, it is not private anymore. This consideration is not holding if we accept the view that rules are in a state of constant renegotiation (i.e., if it is shared, it should be changed to be a private again). So, if the private rules are to be renegotiated, how the algorithmic rules stay the same? The tension here consists of the following contradiction: the algorithm which is not yet used, or deployed, describe the fictional, imaginary world. Once running, executed, deployed or used, the algorithm may describe the real state of affairs as is or as it should be (i.e., rules and numbers mirror or not the actual existing reality vs private and shared dichotomy). Another tension to bring up is about an algorithm that is not yet actually running, executed or deployed but only in the state of the simulation or during the 
process of modeling. We would defend the position that for an algorithm to be run or executed, the real action of an individual must take place. Thus, modeling and simulation are mostly unrealized.

Leaving aside above tensions, let us consider the difference between meaning and interpretation of the rules as a convention. The meaning of a rule is not only a matter of its verbal interpretation and acting according to the rule is not always the same as following the rule. Understanding the rule implies that the rule is correctly, consistently and coherently applied in diverse circumstances through various techniques. The application of the rule is then the question of a prolonged practice and judgment. Let us assume the simplest case where one follows a rule if a person is able to justify actions in accordance with the rule. But we consider only the ex-ante and not ex-post case and an imagined action for the existing rule would not be acceptable. We also note that for a given rule and accompanied description, their future interpretation could be construed in such ways that the future action based on that rule could be considered correctly or not.

To sum up, combining rules and numbers leave us with plenty of conditions under which the claim 'reasonable doubt is impossible' might occur on very rare occasions: a reasonable doubt about the future action not yet occurred is hard to come by except in dreams and fantasies that are all doubtful while infused with rules and numbers represented as signs and symbols.

We finish this case by illustrating how the story could unfold for Azeus and Begoa. Firstly, Azeus holds a very strict and narrow view on the notion of the algorithm as numbers in the ordinary sense. Moreover, Azeus does not share it, nor write it down or leave other perceivable marks of. Secondly, 
Azeus imagines an algorithm as rule, as a sequence of symbols and keeping them secret. Once starting executing actions, the algorithm may be deceiving: imagined action might differ from the realized one. Then Azeus may have difficulty justifying just occurred action to Begoa as rules are not private anymore, nor easy or obvious to explain beforehand.

Shared issues and numbers (second case)

Let us consider a second case and feature of moral certainty, namely "everyone seems to know the issue in question" (Hermann 2015). Let us look firstly into numbers and remember that we consider exante state of the algorithm. The formulation of this criterion needs some clarification. In present conditions, "everyone" means only one individual and the 'every' is not other people, but only one person we dare sharing with our experience. Leaving aside this constraint, we can say that the moral issue for one person may not be the issue at all for another. Moreover, the fact of knowing an issue necessitates a moral reasoning beforehand and does not imply that the issue will stay the same in the future. Moreover, moral reasoning requires handling of moral knowledge which we exclude: moral certainty is toward action and moral knowledge is about epistemic claims and reasoning toward or based on these actions (Whittaker 2009).

Considering algorithm as combination of rules and numbers poses an apparent contradiction with a previous case when we accepted the existence of private rules. However, we can analyze only the number, algorithm and moral issues. The problem becomes apparent if we remember that the meaning and interpretation in the case of mathematical operation represented as symbols are very similar in 
some circumstances. But this does not imply that the moral issues understood as the choice between right and wrong, good and bad, follow the same reasoning. In short, we face eventual contradictions.

For illustrating the second case, Begoa may not be aware of the issue that Azeus is facing as Azeus stays silent about. Moreover, Begoa is not aware of the rules imagined by Azeus and not yet shared. As for the algorithm as number, Begoa may consider mathematical operations as morally neutral. Seemingly oblivious to surrounding, Azeus is confined to solitude.

\section{Doubt and common sense (third case)}

Let us consider the third criterion, namely, "attempts to doubt suggest a lack of common sense". Following Boncompagni (2016, 134-136), we adopt a position that the common sense is the combination of holism and the community where practice and action play a foundational role. Accordingly, a common sense may be absent in the case of a solitary person but let us suppose the existence of a companion. Let us consider the case whether it makes sense to doubt rules in the imagined future (i.e., an aspirational algorithm). First, the tension becomes apparent if we think about private and future rules that are not yet shared with the already shared one: that fact of sharing a private rule makes it disappear from the future and not private at all. A second tension arises alongside the wished and the fulfilled aspirations (i.e., an algorithm as to be realized vs. as actual or used). In the case of an algorithm as aspiration, once our wishes are realized, we may avoid sharing them with others because private aspiration are not private anymore, even if we are satisfied with them. Otherwise, if 
conceived algorithm turns out deceiving us, sharing it would mean choosing to be, for example, resentful with others (i.e., selecting between bad and wrong, good and right).

In the third case, it makes sense for Azeus to avoid doubting about imaginary futures. The private algorithm is not private anymore once trying to speak about to Begoa, nor it is in the future anymore. If the private algorithm corresponds fully to initial wishes, Azeus may avoid informing Begoa. Otherwise, Azeus may seem to be resentful once deceived by imagined algorithm and still wanting to share it.

\section{Unanticipated mistake (fourth case)}

Let us consider the fourth case, "mistakes are impossible" (Hermann 2015). Before delving into, we clarify the notion of a mistake as the error in judgment involving choice and decision by describing the most idealized circumstances when and where a mistake is nearly impossible, very unlikely or a mere accident. If the rules and numbers are private and belong to one individual, then their meanings and interpretations may coincide with each other. Making a mistake in such situation necessitate a comparison, extension, or analogy for the imagined and future algorithm. But such mistakes in mathematical operations make no sense and contradict a common sense (see previous case). Moreover, justification of mathematical operations and of ethical reasoning differ greatly. For example, Gill (2019, 204-205) notes that "ethical justification does not involve the formal premises and regimented theories that mathematics does". The contradiction here is that a mathematical practice and principles forbid mistakes and errors while computational operations and techniques reveal them. Another circumstance to consider is when and where the tension is between the algorithm as wished for and as 
fulfilling our intention. In such circumstances, unanticipated mistakes, the ones committed in reasoning or judgment and once confined to aspiration, becomes evident. Yet another difficulty arises when we consider private and shared duopoly which was previously described.

For this fourth case, Azeus imagines dreams full of actions and without contradictions or paradoxes. This dream may fall apart once reasoning and judgment are considered. Moreover, while trying to think about mathematical symbols and operations, Azeus may not realize mistakes and errors as such until the real and actual ones occur.

\section{Additional reason and certainty (fifth case)}

Let us consider the fifth case, the one dealing with "no supporting reasons that are more certain" (Hermann 2015). We note that 'reason' here, as we understand it, refers to judgment and reasoning in the moral sense. For the sake of the argument, we accept that 'reason' may not mean reasoning and logical inference which automatically exclude this criterion from our case by making it more appropriate for epistemic claims in traditional sense (i.e., intentional cognitive efforts), but that they were executed and memorized in the past and became a routinized habit partially due to the algorithmic use. Set aside this note, we could ask then about the circumstances in which more certain moral givens would not change the claim. First, we consider the algorithm as aspiration. We admit that more certain facts will not change too much the overall picture if the algorithm stays private. However, if the algorithm is shared, it is already realized or, at least, not imagined anymore: in the case of simulations and models, we could imagine an additional circumstance more certain than before. Secondly, let us 
consider a following example: algorithm as wished and an algorithm as fulfilling our hopes. We could argue that it is possible to imagine an idealized situation where and when realized algorithm could satisfy our expectation, but we could provide an additional and more certain circumstances in our heads. This could hold for an algorithm as wished for and an algorithm as realized. In the latter case, an additional issue arises, namely the challenge of imaging a future less certain than the previous one.

For the fifth case, Azeus keeps a private algorithm secret and hardly fantasizes more perfect futures. Moreover, this private algorithm could help to articulate a substantially more impeccable future. However, once faithfully following this algorithm, Azeus could invent yet another justification for the future. Such imagined actions may fall under the category called by DeSouza (2013) “pre-reflective ethical know-how".

Inarticulate certainty (sixth case)

Before investigating last case, we clarify the criterion "certain cannot be meaningfully uttered in ordinary context" (Hermann 2015). Shortly written, the certainty is about something that could not be expressed, communicated, even to ourselves, nor it is an emotion or feeling (Lupyan and Winter 2018; Zdrazilova et al. 2018). We add that 'uttered' here may mean unsayable, impossible to articulate as a sound or corporal gesture that may become a word or habit. As such, it may have meaning and might be interpreted in various ways. It may also denote the general frontier or a horizon between eventually intelligible and not, between perception and void. In other terms, the certainty is about fleeing moments that are just arrived and comes from the future. 
Let us consider firstly an algorithm as aspiration. As imagined in the future, an algorithm may show all the signs of the unsayable and as frontier because numbers and mathematical proofs suggest the absence of contradictions and paradoxes. In the case of the algorithm viewed as numbers, this case may be moot. But in the case of rules, an algorithm may start dissolving once certainty is questioned as the rules and instructions suppose some degree of interpretation and fuzziness in regard to the envisioned actions. A second situation touches upon a point of passage between an algorithm as imagined and as realized (i.e., ex-ante vs. ex-post). The blurry line between an algorithm as wished and algorithm as not fulfilled could make it plausible that algorithm is indistinguishable but still certain to some degree. Finally, if the algorithm is as future expectation, then our imagination is its limits, whereas in the present, it must come from something else, usually the existence of the other.

As the illustration of the sixth case, Azeus whispers or mumbles some sounds or scrabbles some signs which, if pressed, could not be coherently articulated but still certain about. Moreover, Azeus could be overwhelmed and feeling as losing ground or sense of reality while, once asked, this realization is not of so much help.

\section{Summing up}

To sum up all six cases, we enumerate moral tensions potentially leading to moral issues and problems: (a) the choice between sharing a private algorithm with others and a realized one; (b) the choices between an aspirational algorithm and realized and wished for; (c) the selection between an algorithm 
as really fulfilling our expectations and algorithm as future unrealized hope combined with dichotomy private and shared.

The delicate balance alongside the future, the present as hoped for and as factual and lived gives us following situations: be suspicious of others while credulous of rules (doubt and future: first case); be oblivious and alone (shared issues and numbers : second case); be often lonely and impulsive (doubt and common sense : third case); be overconfident and pedantic (unanticipated mistake : fourth case); be rigid and stay in the frozen present (additional reasons and certainty : fifth case); be inaccessible: choosing between mirage of the future and the fog of the present detached from reality (inarticulate certainty : sixth case).

\section{Implications}

Among implications, we highlight the following. Firstly, algorithmic opacity is hard to detect in advance. This does not mean that we could not contemplate some version of algorithmic opacity based on the analogy or similarity, but trade-offs between competing characteristics underpinned by action may make it difficult: choosing between correct and good depends usually on the agreements, communities and practices. A previously unknown and novel kinds of opacity are hard to anticipate and only the old ones from (observation of empirical) past could be projected into future (see fourth case). While being part of newly created algorithm, errors and mistakes that may be imagined, have their sources in the present or the past. This may also include mathematical proofs and definitions (Gorroochurn 2011) as well as computational operations and artifacts (Smith 1993; Fresco and 
Primiero 2013). Thus, if algorithms, as a part of digital lives of the majority in some society, break down existing social institutions, conventions, and rules, they do not necessarily promote the creation of the new ones, nor even facilitate the replacement of them; at best, algorithms shatter existing social arrangements while hindering their substitution with the new one by creating conditions inhibiting (public) conversations and a wide consensus in shared spaces. Moreover, if thinking about the future means mainly reflecting about future possibilities, then it is hard to imagine a case when and where such fictions and products of imagination might differ. Thus, their fantastic character and immaculate features are still in the same category until the reality and time touch upon them.

Secondly, the pivotal moment in the story of algorithmic opacity is the point of passage between imagined future and the realized present. This moment of algorithmic executions, deployments and persistent uses is the moment when opacity may become apparent. This moment is the nexus point between expected future and just occurred present.

Thirdly, moral issues will not always lead to moral problems for self or others while moral tension may still be there. Simply stated, when future private expectations as imagined by one person do not diverge from actual algorithmic functioning, moral tensions may not lead to issues, nor problems. We could call this situation dormant issues and eventual problems. For example, we are satisfied with the algorithmic logic because when we expect to type a word on the computer keyboard, this or that word appears on the screen, we just do not expect otherwise. Inversely, we may not share with others the algorithm and some issues may be dormant for a long time and may become a problem once a minor change just occurs. 
Furthermore, issues may become problems when and where disharmony and dissonance between our expectations of algorithmic behavior, its actual behavior and our own wishes to act are inexorably evident. Inversely, when and where future expectations of actions, realized expectations and our lived experience do not differ from each other is the case of almost absent algorithmic opacity. In other words, algorithmic opacity and its absence are not only a matter of sensations, perceptions.

Finally, algorithmic opacity may help to mitigate the black-box problem if moral certainty is properly understood and handled alongside other considerations. Let us imagine a following plot. If we consider and accept that feeling and emotion somehow influence behaviors, and everyday actions by affecting our motivation and attention, we can imagine a scenario when and where the mood, a feeling of being bored or disgusted affect decision and cloud reasoning. In some situation, such negative emotions may influence the grasp of the meaning and interpretation of envisioned actions.

\section{Conclusion}

Algorithms play an important role in our lives. While offering a multitude of benefits (e.g., the convenience of keeping in touch with friends and family, organizing our lives and simplifying everyday routines), algorithms still remain an obscure and enigmatic object to live with (e.g., the issues of fairness, accountability). One of their persistent features is an algorithmic opacity understood as the absence to various degrees of our comprehension and understanding of how algorithms work, how they obtain results that are exact, precise, accurate, and trustworthy (i.e., the 'black-box problem'). In this 
essay, we try to elucidate the question of algorithmic opacity and moral certainty from the individual perspective by following virtue ethic tradition.

For clearly articulating our position in this debate, we need to consider three premises summarized as the following. Firstly, an algorithm is understood as rules and numbers for simplifying our lives and sharing with others. Secondly, the opacity is distinct from knowledge and physical features of the world. Thirdly, as resisting the urge to doubt, moral certainty is non-epistemic and foundational condition for action. Then our argument consists of two statements and conclusion: (I) an algorithm is anticipatory aspirations before its executions, deployments or uses and (II) once running, deployed or used, an algorithm as anticipation of future events and activities may become (a) realized and presently concrete or, (b) lacking or missing our expectation. As the inherent feature of an algorithm, algorithmic opacity is based therefore on moral tensions, issues and problems all linked to action imagined or realized.

This essay can be situated in the larger context for those who are interested in platforms and infrastructural investigations including digital and globe-spanning ones. Through the work of Mackenzie (2019), who defines a platform as technical procedures ready-to-use and widely diffused and platform as abstracted intersection for different groups, we can advance that the notion of an algorithm articulated here could serve as a glue between platform and infrastructure. Specifically, algorithmic opacity might be considered as a boundary for a platform and constraint for infrastructure: in the former case, platformization facilitates diffusion of a particular algorithm which, by its own 
logic, engenders tensions and issues for the growth of a platform on one side and, on the other, inhibits the formation of infrastructural teams.

The work of Fisher and Mehozay (2019) offers another example where the interests and focus are closer if not overlapping. Their goal is to sketch out an algorithmic episteme of digital media and to contrast it against a previous version of traditional mass media. According to them, algorithmic episteme is performative (i.e., "what human are" and predicted action) and rests on two assumptions, namely (a) digital footprint and observed behavior, and (b) superficial features and characteristics overwhelming others (e.g., frivolous and insignificant vs. interpretations and justifications based upon long-practiced reasoning, conversations, and dialogues). Although we would agree with Fisher and Mehozay that the essentialist version of social categories are drowned out and ultimately precluded in modern digital media platforms, we would like to highlight two points in regard to their assumptions. First, it is usually assumed that data of digitized events are accurate and precise (i.e., in data-science parlance two corresponding words are bias and variance). Second, the observation of behavior (i.e., “digitally registered behavior") will tell something (i.e., "more data is better" principle). Both of these points are undermined in the case of algorithmic opacity as described earlier: observed events may not tell anything at all about what is going on in the best circumstances, or, other extreme, they may lead in the wrong direction over a prolonged period of time may (i.e., a distortion of prediction).

Although we only explored an individual standpoint, algorithm may be best considered as the combination of three distinct perspectives: individual, group and whole society. Following recent mention by Lee and Larsen (2019) to investigate algorithm through the lens of 'hood', we tried to 
enrich existing conceptual vocabulary and schemata by considering an algorithm as the hood from firstperson view, individualistic standpoint and without considering two others. This opens the door for proper investigation of the individual behavior and character (i.e., a various kinds of personhood such as petienthood (Joyce et al. 2020)). In such case, our personal algorithms also affect others during the day-to-day usual routines (i.e., neighborhood: workplace, leisure, culture, and entertainment). Lastly, our algorithms could affect the sense of belonging and allegiance on the national and state level (i.e. statehood) if legitimacy is brought to the picture.

To conclude, we remark the following. By posing the question of algorithmic opacity and moral certainty, we are widening the debate about algorithmic conceptual schemata (i.e., a grammar, vocabulary, and rules) available to the general public, professionals, and experts for properly considering role, function and place of an algorithm in digitized social realm. In other words, for speaking and deliberating meaningfully about problems related to algorithms, we need new cognitive categories and appropriate conceptual boundaries (Spillman 2020).

\section{Acknowledgment}

Author is thankful to Prof. Dominic Martin (ESG, UQAM, Montreal, Canada) for insightful comments and suggestions on previous version of this manuscript. Author is grateful to the participants and panelists of EASST/4S Conference, virtPrague, $20^{\text {th }}$ August for productive conversations. Author declares no conflict of interest. 
References:

Abasnezhad, A., \& Jenkins, C. S. I. 2018. "Metaphysical Vagueness Without Vague Objects." Thought: A Journal of Philosophy, 7(4), 278-283. https://doi.org/10.1002/tht3.398

Abbott, O. 2020. The Self, Relational Sociology, and Morality in Practice. Cham, Switzerland: Palgrave Macmillan. https://doi.org/10.1007/978-3-030-31822-2

Abend, G. 2019. "Moral decisionism and its discontents." Journal for the Theory of Social Behaviour, 49(1), 59-83. https://doi.org/10.1111/jtsb.12191

Åkerman, J. 2012. “Contextualist Theories of Vagueness.” Philosophy Compass, 7(7), 470-480. https://doi.org/10.1111/j.1747-9991.2012.00495.x

Azzouni, J. 2017. "How Positive Success Curves Enable Private Rule Following." In The RuleFollowing Paradox and its Implications for Metaphysics. Cham: Springer International Publishing. https://doi.org/10.1007/978-3-319-49061-8_5

Bailey, O. 2017. "What Knowledge is Necessary for Virtue?" Journal of Ethics and Social Philosophy, 4(2), 1-18. https://doi.org/10.26556/jesp.v4i2.40

Beckert, J. 2019. "Markets from meaning: quality uncertainty and the intersubjective construction of value." Cambridge Journal of Economics, 44(2), 285-301. https://doi.org/10.1093/cje/bez035

Bernoulli, J., Bernoulli, J., \& Sylla, E. D. 2006. The Art of Conjecturing, Together with Letter to a Friend on Sets in Court Tennis. Baltimore, USA: Johns Hopkins University Press.

Binns, R. 2019. "Human Judgement in Algorithmic Loops; Individual Justice and Automated DecisionMaking." SSRN Electronic Journal. https://doi.org/10.2139/ssrn.3452030

Bogosian, K. 2017. “Implementation of Moral Uncertainty in Intelligent Machines.” Minds and Machines, 27(4), 591-608. https://doi.org/10.1007/s11023-017-9448-Z

Boncompagni, A. 2016. “Chapter 3: Common Sense and Weltbild.” In Wittgenstein and Pragmatism: On Certainty in the Light of Peirce and James. London, UK: Palgrave Macmillan UK. https://doi.org/10.1057/978-1-137-58847-0_4

Brundage, M. 2014. "Limitations and risks of machine ethics." Journal of Experimental \& Theoretical Artificial Intelligence, 26(3), 355-372. https://doi.org/10.1080/0952813X.2014.895108 
Cath, C. 2018. "Governing artificial intelligence: ethical, legal and technical opportunities and challenges." Philosophical Transactions of the Royal Society A: Mathematical, Physical and Engineering Sciences, 376(2133), 20180080. https://doi.org/10.1098/rsta.2018.0080

Chabert, J. L., Weeks, C., Barbin, E., Borowczyk, J., Chabert, J. L., Guillemot, M., Michel-Pajus, A., Djebbar, A., \& Martzloff, J. C. 1999. A History of Algorithms: From the Pebble to the Microchip. Berlin, Germany: Springer Berlin Heidelberg. https://doi.org/10.1007/978-3-642$\underline{18192-4}$

Clark, A. 2015. Surfing Uncertainty: Prediction, Action, and the Embodied Mind. New York, NY, USA: Oxford University Press.

Coliva, A. 2016. "Which Hinge Epistemology?" International Journal for the Study of Skepticism, 6(23), 79-96. https://doi.org/10.1163/22105700-00603002

Curren, R. 2019. "Wisdom and the Origins of Moral Knowledge." In Virtue Ethics: Retrospect and Prospect, edited by E. Grimi, 67-80. Cham: Springer International Publishing. https://doi.org/10.1007/978-3-030-15860-6 6

Danaher, J. 2019. "The rise of the robots and the crisis of moral patiency." AI \& SOCIETY, 34(1), 129136. https://doi.org/10.1007/s00146-017-0773-9

De Mesel, B. 2015. "Moral arguments and the denial of moral certainties." In Realism-relativismconstructivism. Papers of the 38th international Wittgenstein symposium. August 9-15, 2015 (Vol. XXIII), edited by C. Kanzian, J. Mitterer, \& K. Neges, 68-70. Kirchberg am Wechsel: Austrian Ludwig Wittgenstein Society. https://www.alws.at/alws/wp-content/uploads/2018/06/papers-2015.pdf\#page=69

De Mesel, B. 2018. "How Morality Can Be Absent from Moral Arguments." In The Later Wittgenstein and Moral Philosophy. Cham: Springer International Publishing. https://doi.org/10.1007/978-3$\underline{319-97619-8 \quad 8}$

DeSouza, N. 2013. "Pre-Reflective Ethical Know-How." Ethical Theory and Moral Practice, 16(2), 279-294. https://doi.org/10.1007/s10677-012-9333-z

Diwekar, U., Amekudzi-Kennedy, A., Bakshi, B., Baumgartner, R., Boumans, R., Burger, P., Cabezas, H., Egler, M., Farley, J., Fath, B., Gleason, T., Huang, Y., Karunanithi, A., Khanna, V., Mangan, A., Mayer, A. L., Mukherjee, R., Mullally, G., Rico-Ramirez, V., Shonnard, D., Svanström, M., $\&$ Theis, T. 2021. "A perspective on the role of uncertainty in sustainability science and engineering." Resources, Conservation and Recycling, 164, 105140.

https://doi.org/10.1016/j.resconrec.2020.105140 
Durán, J. M., \& Formanek, N. 2018. “Grounds for Trust: Essential Epistemic Opacity and Computational Reliabilism." Minds and Machines, 28(4), 645-666. https://doi.org/10.1007/s11023-018-9481-6

Ervas, F., Ledda, A., Ojha, A., Pierro, G. A., \& Indurkhya, B. 2018. "Creative Argumentation: When and Why People Commit the Metaphoric Fallacy." Frontiers in Psychology, 9(1815), 1815. https://doi.org/10.3389/fpsyg.2018.01815

Espeland, W., \& Yung, V. 2019. "Ethical dimensions of quantification." Social Science Information, 58(2), 238-260. https://doi.org/10.1177/0539018419851045

Feest, U. 2017. "Phenomena and Objects of Research in the Cognitive and Behavioral Sciences." Philosophy of Science, 84(5), 1165-1176. https://doi.org/10.1086/694155

Finn, E. 2019. "The Black Box of the Present: Time in the Age of Algorithms." Social Research: An International Quarterly, 86(2), 557-579. https://muse.jhu.edu/article/732190

Fisher, E., \& Mehozay, Y. 2019. "How algorithms see their audience: media epistemes and the changing conception of the individual." Media, Culture \& Society, 41(8), 1176-1191. https://doi.org/10.1177/0163443719831598

Fresco, N., \& Primiero, G. 2013. “Miscomputation.” Philosophy \& Technology, 26(3), 253-272. https://doi.org/10.1007/s13347-013-0112-0

Gill, M. B. 2019. "Morality is Not Like Mathematics: The Weakness of the Math-Moral Analogy." The Southern Journal of Philosophy, 57(2), 194-216. https://doi.org/10.1111/sjp.12322

Gorroochurn, P. 2011. "Errors of Probability in Historical Context." The American Statistician, 65(4), 246-254. https://doi.org/10.1198/tas.2011.11146

Green, B. 2019. “The Essential Ambiguity of the Social.” Philosophy of the Social Sciences, 49(2), 108-136. https://doi.org/10.1177/0048393118821185

Guizzardi, G. 2020. "Ontology, Ontologies and the "I" of FAIR.” Data Intelligence, 2(1-2), 181-191. https://doi.org/10.1162/dint_a 00040

Hagendorff, T., \& Wezel, K. 2019. “15 challenges for AI: or what AI (currently) can’t do.” AI \& SOCIETY, 35(2), 355-365. https://doi.org/10.1007/s00146-019-00886-y

Hansen, L. K., \& Rieger, L. 2019. “Interpretability in Intelligent Systems - A New Concept?” In Explainable AI: Interpreting, Explaining and Visualizing Deep Learning, edited by W. Samek, G. Montavon, A. Vedaldi, L. K. Hansen, \& K.-R. Müller, 41-49. Cham: Springer International Publishing. https://doi.org/10.1007/978-3-030-28954-6 3 
Hermann, J. 2015. On Moral Certainty, Justification and Practice : A Wittgensteinian Perspective. Hampshire, UK: Palgrave Macmillan. https://doi.org/10.1057/9781137447180

Herzog, C. 2019. "Technological Opacity of Machine Learning in Healthcare.” In Weizenbaum Conference 2019 "Challenges of Digital Inequality - Digital Education, Digital Work, Digital Life", 1-9. Berlin, Germany: SSOAR. https://doi.org/10.34669/wi.cp/2.7

Hey, J. D., Neugebauer, T. M., \& Pasca, C. M. 2010. “Georges-Louis Leclerc de Buffon's 'Essays on Moral Arithmetic'." In The Selten School of Behavioral Economics: A Collection of Essays in Honor of Reinhard Selten, edited by A. Sadrieh \& A. Ockenfels, 245-282. Heidelberg, Germany: Springer Berlin Heidelberg. https://doi.org/10.1007/978-3-642-13983-3_ 15

Hill, R. K. 2016. “What an Algorithm Is.” Philosophy \& Technology, 29(1), 35-59. https://doi.org/10.1007/s13347-014-0184-5

Hoyos-Valdés, D. 2018. "The notion of character friendship and the cultivation of virtue." Journal for the Theory of Social Behaviour, 48(1), 66-82. https://doi.org/10.1111/jtsb.12154

Høyrup, J. 2018. "When is the algorithm concept pertinent-and when not? Thoughts about algorithms and paradigmatic examples, and about algorithmic and non-algorithmic mathematical cultures." Mathematics, 3(1), 211-232. https://doi.org/10.3934/Math.2018.1.211

Joyce, K. A., James, J. E., \& Jeske, M. 2020. "Regimes of Patienthood: Developing an Intersectional Concept to Theorize Illness Experiences." Engaging Science, Technology, and Society, [S.l.], 6, 185-192. https://doi.org/10.17351/ests2020.389

Kampourakis, K., \& McCain, K. 2019. Uncertainty: How It Makes Science Advance. New York, NY, USA: Oxford University Press.

Katz, O., \& Shalev Greene, K. 2020. “Constructing time in uncertainty: Temporal regimes among missing persons’ families.” Current Sociology, O(0), 0011392120902235. https://doi.org/10.1177/0011392120902235

Keil, G. 2013. "Introduction: Vagueness and Ontology.” Metaphysica, 14(2), 149-164. https://doi.org/10.1007/s12133-013-0118-1

Kessler, O. 2008. "Uncertainty, Rationality and the Study of Social Institutions." Review of Social Economy, 66(4), 501-522. https://doi.org/10.1080/00346760801932692

Kroll, J. A. 2018. "The fallacy of inscrutability." Philosophical Transactions of the Royal Society A: Mathematical, Physical and Engineering Sciences, 376(2133), 20180084. https://doi.org/10.1098/rsta.2018.0084 
Krzychała, S. 2019. "Professional Praxis Community in a Dialogical Perspective: Towards the Application of Bakhtinian Categories in the Documentary Method." Forum Qualitative Sozialforschung / Forum: Qualitative Social Research, 20(1). https://doi.org/10.17169/fqs$\underline{20.1 .3073}$

Lee, F., \& Björklund Larsen, L. 2019. "How should we theorize algorithms? Five ideal types in analyzing algorithmic normativities.” Big Data \& Society, 6(2), 2053951719867349. https://doi.org/10.1177/2053951719867349

Lupyan, G., \& Winter, B. 2018. "Language is more abstract than you think, or, why aren't languages more iconic?” Philosophical Transactions of the Royal Society B: Biological Sciences, 373(1752), 20170137. https://doi.org/10.1098/rstb.2017.0137

Lynch, M. 2014. “At the margins of tacit knowledge.” Philosophia Scientice, 17(3), 55-73. https://dx.doi.org/10.4000/philosophiascientiae.886

Mackenzie, A. 2019. "From API to AI: platforms and their opacities." Information, Communication \& Society, 22(13), 1989-2006. https://doi.org/10.1080/1369118X.2018.1476569

Malin, B. J. 2019. "Contextual Materialism: Mereology, Sociality and the Vague Ontology of Media Objects.” Communication Theory, 29(2), 151-168. https://doi.org/10.1093/ct/qty034

Martignon, L. 2015. “Algorithm.” In International Encyclopedia of the Social \& Behavioral Sciences (Second Edition), edited by J. D. Wright, 529-533. Amsterdam, Netherlands: Elsevier Ltd. https://doi.org/10.1016/B978-0-08-097086-8.43002-3

Merricks, T. 2001. "Varieties of Vagueness." Philosophy and Phenomenological Research, 62(1), 145157. https://doi.org/10.1111/j.1933-1592.2001.tb00045.x

Misselhorn, C. 2018. “Artificial Morality. Concepts, Issues and Challenges.” Society, 55(2), 161-169. https://doi.org/10.1007/s12115-018-0229-y

Mittelstadt, B. 2019. "Principles alone cannot guarantee ethical AI." Nature Machine Intelligence, 1(11), 501-507. https://doi.org/10.1038/s42256-019-0114-4

Mittelstadt, B. D., Allo, P., Taddeo, M., Wachter, S., \& Floridi, L. 2016. "The ethics of algorithms: Mapping the debate." Big Data \& Society, 3(2), 2053951716679679. https://doi.org/10.1177/2053951716679679

Moulin-Stożek, D. 2019. "The social construction of character." Journal for the Theory of Social Behaviour, 49(1), 24-39. https://doi.org/10.1111/jtsb.12188

Moyal-Sharrock, D. 2004. "Linguistic and Personal Hinges." In Understanding Wittgenstein's On Certainty. Hampshire, UK: Palgrave Macmillan UK. https://doi.org/10.1057/9780230504462 7 
Moyal-Sharrock, D., \& Brenner, W. (2005). Readings of Wittgenstein's On Certainty. Hampshire, UK: Palgrave Macmillan UK.

Neyland, D. 2015. “On Organizing Algorithms.” Theory, Culture \& Society, 32(1), 119-132. https://doi.org/10.1177/0263276414530477

Nyholm, S. 2018. "The ethics of crashes with self-driving cars: A roadmap, I." Philosophy Compass, 13(7), e12507. https://doi.org/10.1111/phc3.12507

O’Hara, N. 2018. "Basic Certainty and Morality." In Moral Certainty and the Foundations of Morality. Cham: Springer International Publishing. https://doi.org/10.1007/978-3-319-75444-4_1

Ogawa, T. 2001. "A review of the history of Japanese mathematics." Revue d'histoire des mathématiques, 7(1), 137-155. http://www.numdam.org/item/RHM_2001_7_1_137_0

Poli, R., \& Obrst, L. 2010. "The Interplay Between Ontology as Categorial Analysis and Ontology as Technology." In Theory and Applications of Ontology: Computer Applications, edited by R. Poli, M. Healy, \& A. Kameas, 1-26. Dordrecht: Springer Netherlands. https://doi.org/10.1007/978-90-481-8847-5 1

Roberge, J. 2011. "What is critical hermeneutics?" Thesis Eleven, 106(1), 5-22. https://doi.org/10.1177/0725513611411682

Romele, A., Severo, M., \& Furia, P. 2020. "Digital hermeneutics: from interpreting with machines to interpretational machines." AI \& SOCIETY, 35(1), 73-86. https://doi.org/10.1007/s00146-018$\underline{0856-2}$

Russell, D. C. 2013. The Cambridge Companion to Virtue Ethics. Cambridge, UK: Cambridge University Press.

Saarinen, R. 1993. "John Buridan and Donald Davidson on "Akrasia"." Synthese, 96(1), 133-153. https://doi.org/10.1007/BF01063806

Schönbaumsfeld, G. 2016. “'Hinge Propositions' and the 'Logical' Exclusion of Doubt.” International Journal for the Study of Skepticism, 6(2-3), 165-181. https://doi.org/10.1163/22105700$\underline{00603006}$

Shadbolt, N., O’Hara, K., De Roure, D., \& Hall, W. 2019. "Privacy, Trust and Ethical Issues.” In The Theory and Practice of Social Machines, edited by N. Shadbolt, K. O'Hara, D. D. Roure, \& W. Hall, 149-200. Cham: Springer International Publishing. https://doi.org/10.1007/978-3-03010889-2 4

Shapiro, S. 2006. Vagueness in Context. Oxford, UK: Oxford University Press. 
Shin, D., \& Park, Y. J. 2019. "Role of fairness, accountability, and transparency in algorithmic affordance." Computers in Human Behavior, 98, 277-284.

https://doi.org/10.1016/j.chb.2019.04.019

Smith, B. C. 1993. "Limits of Correctness in Computers." In Program Verification: Fundamental Issues in Computer Science, edited by T. R. Colburn, J. H. Fetzer, \& T. L. Rankin, 275-293. Dordrecht: Springer Netherlands. https://doi.org/10.1007/978-94-011-1793-7_13

Spillman, L. 2020. What is Cultural Sociology? Cambridge, UK: Polity Press.

Sriram, M. S. 2005. "Algorithms in Indian Mathematics." In Contributions to the History of Indian Mathematics, edited by G. G. Emch, R. Sridharan, \& M. D. Srinivas, 153-182. Gurgaon: Hindustan Book Agency. https://doi.org/10.1007/978-93-86279-25-5 7

Tahko, T. E. 2012. "Boundaries in Reality.” Ratio, 25(4), 405-424. https://doi.org/10.1111/j.14679329.2012.00554.X

Taylor, C. 1980. "Understanding in Human Science." The Review of Metaphysics, 34(1), 25-38. http://www.jstor.org/stable/20127456

Taylor, C. 1985a. Philosophical Papers: Volume 1, Human Agency and Language. Cambridge, UK: Cambridge University Press.

Taylor, C. 1985b. Philosophical Papers: Volume 2, Philosophy and the Human Sciences. Cambridge, UK: Cambridge University Press.

Totaro, P., \& Ninno, D. 2014. "The Concept of Algorithm as an Interpretative Key of Modern Rationality." Theory, Culture \& Society, 31(4), 29-49. https://doi.org/10.1177/0263276413510051

Tripathi, A. K. 2017. "Hermeneutics of technological culture.” AI \& SOCIETY, 32(2), 137-148. https://doi.org/10.1007/s00146-017-0717-4

Vallor, S. 2017. "AI and the Automation of Wisdom." In Philosophy and Computing: Essays in Epistemology, Philosophy of Mind, Logic, and Ethics, edited by T. M. Powers, 161-178. Cham: Springer International Publishing. https://doi.org/10.1007/978-3-319-61043-6_8

Vardi, M. Y. 2012. "What is an algorithm?" Communications of the ACM, 55(3), 5. https://doi.org/10.1145/2093548.2093549

Vertesi, J. 2020. "Testing planets: Institutions tested in an era of uncertainty." The British Journal of Sociology, 71(3), 474-488. https://doi.org/10.1111/1468-4446.12725 
Walker, A. D. M. 1989. "Virtue and Character.” Philosophy, 64(249), 349-362. https://doi.org/10.1017/S0031819100044703

Wasilow, S., \& Thorpe, J. B. 2019. “Artificial Intelligence, Robotics, Ethics, and the Military: A Canadian Perspective." AI Magazine, 40(1), 37-48. https://doi.org/10.1609/aimag.v40i1.2848

Weber, M. A. 2013. "Interrelations and Dissimilarities Between Distinct Approaches to Ontic Vagueness." Metaphysica, 14(2), 181-195. https://doi.org/10.1007/s12133-013-0120-7

Whittaker, J. H. 2009. “R. W. Beardsmore: Understanding Moral Judgement.” In Sense and Reality Essays out of Swansea, edited by J. Edelman, 187-218. Berlin, Germany: Walter de Gruyter GmbH. https://doi.org/10.1515/9783110328813.187

Zdrazilova, L., Sidhu, D. M., \& Pexman, P. M. 2018. "Communicating abstract meaning: concepts revealed in words and gestures." Philosophical Transactions of the Royal Society B: Biological Sciences, 373(1752), 20170138. https://doi.org/10.1098/rstb.2017.0138

Zerilli, J., Knott, A., Maclaurin, J., \& Gavaghan, C. 2019. “Transparency in Algorithmic and Human Decision-Making: Is There a Double Standard?” Philosophy \& Technology, 32(4), 661-683. https://doi.org/10.1007/s13347-018-0330-6 